\title{
Psychological and Social Problems of Hearing-Impaired Students and the Adopted Coping Strategies in Deaf Schools
}

\author{
Dr. Abdellatif Khalaf Suleiman Alramamneh \\ Lecturer, Princess Rahma College, \\ Balqa Applied University, Jordan \\ Dr. Obaid Abdelkarim Obaid Sabayleh \\ Lecturer, Princess Rahma College, \\ Balqa Applied University, Jordan

\section{Dr. Shirin Munier Abdallah Abu hazim \\ Training Supervisor, Princess Rahma College, Balqa Applied University, Jordan} \\ Dr. Samer Mohammed Abu Drei \\ Training Supervisor, Princess Rahma College, \\ Balqa Applied University, Jordan
}

Doi: 10.36941/jesr-2020-0039

\section{Abstract}

The aims of this study were to identify the social and psychological problems of hearing-impaired students and investigate the coping strategies adopted in deaf schools. The descriptive analytical approach was used to describe the data collected from a randomly selected sample that consisted of 150 deaf students; 67 were males and 83 were females. The findings showed that hearing-impaired students face social problems such as difficulties encountered in public places and feeling scared of violence by others. The findings also showed that students with hearing impairment face frequent psychological problems such as explanation of mistakes, fear of making mistakes, and separation anxiety. However, the study results revealed that students with hearing impairment have a medium level of psychological and social problems as well as the adoption level of coping strategies. Furthermore, there are no statistically significant differences on the level of both social and psychological problems, and adapted level of coping strategies due to gender, address, education level, and impairment severity.

Keywords: coping strategies, deaf students, psychological problems, social problems

\section{Introduction}

Hearing is one of the senses, which has a vital role in determining individual life. Hearing has a distinct function of transferring an aspect of the social and environmental components of reality to 
the brain. The significance of hearing sense is demonstrated by the fact that hearing is a source of learning and knowledge acquisition as well as earning direct and indirect experiences. Hearing system transmits a spectrum and an unlimited amount of information about the surroundings. Therefore, hearing impairment affects individuals' cognitive competence, since it reduces the individual's ability to recognize things, particularly the aspects directly related to hearing that reduces his ability to sustain normal activities and processes. Deaf students are known as those individuals who use sign language or lip-reading for communication either using hearing aids or not, and their hearing sensitivity is between 25 and 90 Decibels (dB) (AbuDreea, 2017). The social and psychological problems vary among hearing-impaired students who are born with hearing impairment compared to those who lost their hearing at a late age. However, each problem requires diverse management, services, and training settings, therefore, a teacher must distinguish these problems (ALzahrani, 2007).

Social interaction of hearing-impaired students with their hearing peers reveals various social problems of those students since they do not acquire huge audio information, which has a primary influence on students' social behavior patterns. Consequently, this deficit of information will surely influence their social skills (AbuSha'ban, 2016). In terms of psychological problems, the differences in feeling level among deaf and normal students nourish the anxiety level in deaf students. The closed association among physical deficit of competence and feelings cannot be neglected. Furthermore, hearing impairment may relate directly to the increment of fearing and dependency. A deaf student needs to depend on others to fill the ability deficit from hearing loss, which is considered as a partial dependency, instead, it may turn into a total dependency, and they may lose their self-esteem (Naji \& Qurashi, 2018).

It is worth noting that the common communication language among individuals is useful to foster their relations and understand each other. However, the emotional, behavioral, social, and psychological problems vary among hearing impaired students due to several aspects such as the child age when deafness occurred, the severity of impairment, early diagnosis, the hearing ability of parents, family, and the environmental condition of the child (Alrousan, 2016).

Abu Dreea and Arramamneh (2019) conducted a study to investigate the verbal and performance intelligence and its relation to behavioral problems of hearing-impaired students. The results revealed that hearing-impaired students have a normal intelligence level and a medium level of psychological problems. Moreover, results found that hearing-impaired students show low-level aggression, as well as attention deficit and hyperactivity problems. Ali (2015) investigated the relationship between social competence and social isolation, and he found that there is a statistically significant positive correlation at $\alpha=0.01$ level between social competence and social isolation. It is also found that there are statistically significant differences in social competence level between males and females in favor of female students.

Rimawi (2008) conducted a study to expose the primary psychosocial difficulties that face impaired women in Amman and its relation to impairments categories. The results indicated that impaired women encounter several psychological problems in descending order going from anxiety, depression, isolation, to dependency, in which hearing impaired women have a higher significant level of anxiety compared to other women of impairment, while physically impaired women have a higher significance level of depression among other impaired women. However, there are no differences in social isolation among impaired women. Comparing impaired women to normal women, the differences in anxiety and social isolation were in favor of the impaired women, while there is no difference between them in depression and dependency.

AlA'iq and Yahya (2007) presented a study to identify hearing impaired students' problems from their families and teachers' perspectives in Jordan. The findings support that hearing impaired students are encountering different problems such as behavioral and emotional problems, academic problems, conduct problems, and social problems.

Vanaldik et al, (2009) found that $41 \%$ of children have emotional and behavioral problems in a study on 238 deaf Dutch children aged between 4-10 years, while Dutch children aged between 12-18 
years showed higher level of anxiety, depression, and more social problems compared to those aged between 4-11 years old.

A study conducted by Watson, Neuman , and Dickinson (2001) asserted that both totally and partially hearing-impairing causes deficit in the social development of deaf adolescent, and cause various genre of problems such as aggression, lack of compatibility, anxiety and so forth that obstruct deaf inclusion with others. However, a positive parents' behavior toward these problems eliminates and restricts it. Shirin, Antia, Patricia, and Luckner (2011) who have concerns in social and behavioral skills of teaching deaf and hearing-impaired students in public found that these students have a medium level of social and behavioral problems according to their teachers' perspectives. In the same context, Karen, Kathryn, and Blair (2008) studied the communication and social interaction of hearing-impaired student in American schools. Furthermore, they investigated its impact on the academic success in order to reveal the positive influential variables on the hearing-impaired students' success, and find compatible strategies to manage and solve their communication problems as a consequent. The study results indicated that deaf and hearing-impaired students suffer extremely from conduct and social interaction problems, therefore, researchers advocated designing a training program for communication skills.

\section{$1.1 \quad$ Research Questions}

The study answers the following questions:

1. What are the social problems of Hearing -impaired students?

2. What are the psychological problems of hearing -impaired students?

3. What is the adoption level of coping strategies for social and psychological problems in deaf school?

4. Are there any statistically significant differences among hearing -impaired based on (gender, place of residence, severity of disability, and school grade)?

\subsection{Study Significant}

The study gains significance due to its role in exhibiting the social and psychological problems of hearing-impaired students and exploring the coping strategies for social and psychological problems of hearing-impaired students in schools.

\subsection{Study limitations}

This study has some limitations:

Time limitation: the study is conducted in the first semester of 2019/2020 educational year due to limited access to school complied with mutual consent between educational departments and the researchers.

Spatial limitation: the study is conducted in deaf schools in Salt province only due to researchers' time limitation, spatial divergence among the researchers' province and other provinces, and difficulties of acquiring authorization from relevant educational departments.

Sample profile limitation: the study is conducted on deaf children only who are aged between (6-17) years old in their schools, since these ages are the only one according to records of included schools in the study.

\section{4}

\section{Definitions of Terms}

- Deaf: defined as all individuals who use sign language, or lip reading for communication either using hearing aids or not, and their hearing sensitivity is between 25 and $90 \mathrm{~dB}$. 
- Social Problems: defined as a set of problems and difficulties that hearing-impaired persons faced which cause deficit in their social interaction and communication abilities due to their hearing loss.

- Psychological problems: defined as a set of problems and difficulties that hearing-impaired persons faced which affect their behaviors due to their hearing loss such as anxiety, mood fluctuations, and stress.

- Coping strategies defined as a set of plans and approaches adopted in deaf schools to manage and assess hearing-impaired students' problems.

\section{Methodology: Method and Procedures}

\subsection{Method of study}

This study has used descriptive statistical approach utilizing the quantitative (questionnaire) method as primary tool for data collecting purposes to investigate the research questions.

\subsection{The sample of study}

The study sample purposely selected 150 hearing-impaired students aged between 6 and 17 years old from deaf schools in Salt province in the Hashemite Kingdom of Jordan in order to identify all cases in the study population.

The sample involved 67 male students, and 83 female students corresponding to $45 \%$, 55\% of sample, respectively.

\subsection{The study tool}

\subsubsection{Questionnaire}

A social and psychological problems scale was developed and distributed to hearing -impaired students. The responses of the questionnaire were based on Likert scales ( $5=$ strongly agree, $4=$ agree, $3=$ neutral, 2 =disagree, $1=$ strongly disagree).

The positive statements in the social problems part are the 3, 6, 12, and 14 statements, and in the psychological problems part, they are the 6 and 14, which are respectively weighted as 1,2,3,4, and 5 . This means there are more psychological or social problems when the mean of these responses is large.

As for coping strategies scale, the responses are based on the Likert scale $(5=$ strongly agree, $4=$ agree, $3=$ =neutral, $2=$ disagree, $1=$ strongly disagree). This means if the mean of these responses is large, the use of adaptation strategies becomes greater.

To interpret the responses on each statement of the research questions, the following formula was used:

Range=largest value (strongly agree) - smallest value (strongly disagree)

$=5^{-1}=4$

Class Length $=$ Range $\div$ number of classes

$=4 / 3=1.33$

Thus, the Classes boundaries are:

- From 1 to 2.33 (weak degrees)

- From 2.34 to 3.67 is (mid degrees)

- From 3.68 to 5 is (high degrees) 


\section{$2.4 \quad$ Validity and reliability}

\subsubsection{Social Problems scale}

To investigate the validity of the social problems scale, the coefficient of correlation was calculated between statements and the overall degree of the scale. The validity coefficients ranged from 0.304 to 0.782 . That value indicated the social problems scale was construct validated.

The internal consistency reliability was calculated using the Cronbach's alpha test. The reliability coefficient is 0.901 , which means a suitable value of reliability.

\subsubsection{Psychological Problems scale}

The coefficient of correlation was calculated to investigate the validity of the psychological problems scale. The following table shows the coefficient of correlation between statements and the overall degree of scale:

Table 1: Coefficient of Correlation Between Statements and Overall Degree of Psychological Problems Scale

\begin{tabular}{|c|c|c|c|}
\hline No. statement & Correlation with criterion & No. statement & Correlation with criterion \\
\hline 1 & .669 & 13 & .865 \\
\hline 2 & .731 & 14 & .404 \\
\hline 3 & .341 & 15 & .771 \\
\hline 4 & .562 & 16 & .749 \\
\hline 5 & .681 & 17 & .814 \\
\hline 6 & .374 & 18 & .628 \\
\hline 7 & .758 & 19 & .745 \\
\hline 8 & .541 & 20 & .642 \\
\hline 9 & .817 & & \\
\hline 10 & .794 & & \\
\hline 11 & .863 & & \\
\hline 12 & .835 & & \\
\hline
\end{tabular}

The correlation coefficients between the statements and overall degree of Psychological Problems scale ranged from 0.341 to 0.865 ; that means the Psychological Problems scale was construct validated.

Cronbach's alpha test was used to investigate the internal consistency reliability of this scale. The reliability coefficient is 0.946 ; that means a suitable value of reliability.

\subsubsection{Coping strategies scale}

The coefficient of correlation between statements and overall degree of the scale was calculated; the following table shows these coefficients of the scale:

Table 2: Coefficient of Correlation between Statements and Over All Degree of Coping Strategies Scale

\begin{tabular}{|c|c|c|c|}
\hline No. statement & Correlation with criterion & No. statement & Correlation with criterion \\
\hline 1. & .569 & 15. & .436 \\
\hline 2. & .450 & 16. & .563 \\
\hline 3. & .500 & 17. & .389 \\
\hline
\end{tabular}




\begin{tabular}{|c|c|c|c|}
\hline No. statement & Correlation with criterion & No. statement & Correlation with criterion \\
\hline 4. & .586 & 18. & .447 \\
\hline 5. & .623 & 19. & .333 \\
\hline 6. & .516 & 20. & .380 \\
\hline 7. & .633 & 21. & .414 \\
\hline 8. & .617 & 22. & .338 \\
\hline 9. & .542 & 23. & .340 \\
\hline 10. & .386 & 24. & .586 \\
\hline 11. & .563 & 25. & .402 \\
\hline 12. & .349 & 26. & .489 \\
\hline 13. & .547 & 27. & .452 \\
\hline 14. & .450 & & \\
\hline
\end{tabular}

The correlation coefficients among the statement and overall degree of coping strategies scale ranged from 0,333 to 0.633 ; that means the scale was construct validated which is the test used to determine the extent of measuring the construct supposed to be measured by the scale.

\subsection{Research procedures}

The questionnaire of hearing-impaired students' social and psychological problems and their adaptation strategies level was distributed to hearing -impaired students, and then they filled it with the assistance of helping teachers of the deaf through the following steps: first, the questionnaire was read in sign language by teachers, then the hearing -impaired student has answered the questions, after that the teachers wrote their answer.

The scale was applied by using sign language on 150 hearing-impaired students who are the sample of the study.

The sample of the study was divided into categories depending on gender, age and degree of hearing impairment. After that, the data were processed statistically to find the results, interpret them, and make the recommendations of the study.

\subsection{Data Analysis}

To answer the questions of the study, the Statistical Package of Social Sciences Program (SPSS) was used to process the data after classifying it; the process included:

- Duplicates and percentages to describe the characteristics of the sample of the personal study.

- The Cronbach's alpha test to find the coefficient of reliability.

- The Pearson correlation coefficients to identify the relationship between the social and psychological problems of students with hearing disabilities.

- The arithmetic mean and standard deviations.

\section{The Study Results}

\subsection{What are the social problems of Hearing -impaired students?}

To answer this question, descriptive statistics (means, standard deviations) of the social problems of hearing -impaired students were calculated. The following table showed these results: 
Table 3: Descriptive Statistics (Means, Standard Deviations) of Social Problems of Hearing -impaired Students $(\mathrm{N}=150)$

\begin{tabular}{|c|c|c|c|c|c|}
\hline No & rank & Statements & $\begin{array}{c}\text { standard } \\
\text { deviations }\end{array}$ & Means & level \\
\hline 19 & 1 & I fear of violence from others & 1.076 & 3.58 & middle \\
\hline 7 & 2 & I miss having the privacy in my life & 1.207 & 3.45 & middle \\
\hline 11 & 3 & I miss having the regulation of my free time & .764 & 3.44 & middle \\
\hline 18 & 4 & I have a problem of acceptance of others & 1.030 & 3.41 & middle \\
\hline 10 & 5 & I miss having someone to share my day-to-day problems & 1.217 & 3.36 & middle \\
\hline 9 & 6 & I have a problem with salute and return it & .725 & $3 \cdot 31$ & middle \\
\hline 2 & 7 & I have a problem of opinion expression when I being with hearing peers & 1.316 & 3.25 & middle \\
\hline 16 & 8 & I miss having the self-esteem feeling & .985 & 3.21 & middle \\
\hline 3 & 9 & I make friends with other & 1.416 & 3.21 & middle \\
\hline 21 & 10 & I have a difficulties of acting in public places & 1.433 & 3.18 & middle \\
\hline 8 & 11 & I have a problem of decision acceptance of others & 1.301 & 3.16 & middle \\
\hline 4 & 12 & I'm not participating in any social activity & .712 & 3.15 & middle \\
\hline 6 & 13 & I can communicate with others easily & 1.460 & 3.15 & middle \\
\hline 17 & 14 & I am feeling alone because of the lack of mobility & 1.461 & 3.12 & middle \\
\hline 12 & 15 & I belonged to the surrounding people & 1.313 & 3.03 & middle \\
\hline 13 & 16 & I'm having the trouble of social activities pursuit & 1.359 & 3.01 & middle \\
\hline 5 & 17 & I'm avoiding the parks and public places & 1.196 & 2.99 & middle \\
\hline $\mathbf{1}$ & 18 & I would rather be all alone & 1.154 & 2.75 & middle \\
\hline 14 & 19 & I get compliments and thanks from other & 1.324 & 2.53 & middle \\
\hline 20 & 20 & I have the ability to make different decisions & .844 & 2.14 & low \\
\hline \multirow[t]{2}{*}{15} & 21 & My status within the household less than my brother & .812 & 2.11 & low \\
\hline & & The means of social problems & .679 & 3.07 & middle \\
\hline
\end{tabular}

This table shows that 3.07 is the overall mean of the social problems of Hearing -impaired students, while the means and standard deviations of the statements range between 2.11 to 3.58.

"I fear of violence from others" statement was the highest mean (3.58), while "My status within the household less than my brother" was the lowest (2.11).

\subsection{The second question: What are the psychological problems of hearing -impaired students?}

To answer this question, descriptive statistics (means, standard deviations) of the psychological problems of Hearing -impaired students were calculated. The following table showed these results:

Table 4: Descriptive Statistics (Means, Standard Deviations) of Psychological Problems of Hearing impaired Students $(\mathrm{N}=150)$

\begin{tabular}{|c|c|c|c|c|c|}
\hline No & rank & statements & $\begin{array}{l}\text { standard } \\
\text { deviations }\end{array}$ & Means & level \\
\hline 8 & $\mathbf{1}$ & I do justify when I made any mistake & .708 & 2.81 & middle \\
\hline 9 & 2 & I fear of making mistakes & .734 & 2.77 & middle \\
\hline 2 & 15 & I feel anxious when breaking up with close individuals & .781 & 2.67 & middle \\
\hline 1 & 4 & my personality became introverted & .756 & 2.63 & middle \\
\hline 20 & 5 & I feel inferior because of my inability to attain the basic requirements for life & .746 & 2.56 & middle \\
\hline 4 & 6 & I am ashamed of my disabilities. & .800 & 2.54 & middle \\
\hline 11 & 7 & I'm athwart quickly & .766 & 2.53 & middle \\
\hline 19 & 8 & I am feeling disability because I can't hear & .682 & 2.53 & middle \\
\hline 10 & 9 & I am feeling worried because of the others' contempt for me. & .673 & 2.52 & middle \\
\hline 16 & 10 & I am feeling angry for a trivia & .748 & 2.52 & middle \\
\hline
\end{tabular}




\begin{tabular}{|c|c|l|c|c|c|}
\hline No & rank & statements & $\begin{array}{c}\text { standard } \\
\text { deviations }\end{array}$ & Means & level \\
\hline $\mathbf{1 5}$ & $\mathbf{1 1}$ & It hurts to keep me outside of discussing my family problems. & .663 & 2.49 & middle \\
\hline $\mathbf{1 8}$ & $\mathbf{1 2}$ & I miss feeling happy. & .801 & 2.49 & middle \\
\hline $\mathbf{1 4}$ & $\mathbf{1 3}$ & My parents make me good feelings about my abilities. & .775 & 2.49 & middle \\
\hline $\mathbf{5}$ & $\mathbf{1 4}$ & I'm feeling bad because of the lack of interest about me by others & .730 & 2.47 & middle \\
\hline $\mathbf{7}$ & $\mathbf{3}$ & I have got wild mood swings & .848 & 2.46 & middle \\
\hline $\mathbf{1 2}$ & $\mathbf{1 6}$ & I'm feeling bad because of keeping me away from my relatives & .816 & 2.45 & middle \\
\hline $\mathbf{1 7}$ & $\mathbf{1 7}$ & I am worried about the future & .718 & 2.43 & middle \\
\hline $\mathbf{6}$ & $\mathbf{1 8}$ & I having the self-esteem feeling & .652 & 2.24 & Low \\
\hline $\mathbf{3}$ & $\mathbf{1 9}$ & I have feeling that my parents' love me out of pity & .663 & 2.17 & Low \\
\hline $\mathbf{1 3}$ & $\mathbf{2 0}$ & $\begin{array}{l}\text { My father make me feeling bad because they believe about my abilities } \\
\text { are less than my brothers }\end{array}$ & .739 & 2.13 & Low \\
\hline & & The means of social problems & .520 & 2.50 & middle \\
\hline
\end{tabular}

In this table, 2.50 was the overall mean of the psychological problems of Hearing -impaired students, while the means and standard deviations of the statements ranged between 2.81 and 2.13.

"I do justify when I made any mistake" statement was the highest mean (2.81), while "My father make me feeling bad because they believe about my abilities are less than my brothers" was the lowest (2.13).

3.3 What is the adoption level of coping strategies for social and psychological problems in deaf school?

To answer this question, descriptive statistics (means, standard deviations) of the coping strategies of problems of Hearing -impaired students were calculated. The following table showed these results:

Table 5: Descriptive Statistics (Means, Standard Deviations) of Coping Strategies of The Hearingimpaired Students Problems in Deaf Schools $(\mathrm{N}=150)$.

\begin{tabular}{|c|c|l|c|c|c|}
\hline No & rank & Statements & $\begin{array}{c}\text { standard } \\
\text { deviations }\end{array}$ & Means & level \\
\hline $\mathbf{1 9}$ & $\mathbf{7}$ & The school protects students from violence and abuse. & .594 & 3.42 & middle \\
\hline $\mathbf{2 7}$ & $\mathbf{2}$ & During school time I eliminate the bad traits & .688 & 3.36 & middle \\
\hline $\mathbf{2 6}$ & $\mathbf{3}$ & I am going to move on life because in school & .606 & 3.32 & middle \\
\hline $\mathbf{2 3}$ & $\mathbf{4}$ & During school time my self-confidence became better & .772 & 3.26 & middle \\
\hline $\mathbf{5}$ & $\mathbf{5}$ & $\begin{array}{l}\text { Making pupils aware of hearing disabilities, and their difficulties, } \\
\text { challenges and how to coping it. }\end{array}$ & .788 & 3.13 & middle \\
\hline $\mathbf{9}$ & $\mathbf{6}$ & $\begin{array}{l}\text { The school help the students to decide their destiny and achieve their } \\
\text { independence }\end{array}$ & .917 & 3.07 & middle \\
\hline $\mathbf{8}$ & $\mathbf{1}$ & During school time I felling board & .873 & 3.05 & middle \\
\hline $\mathbf{4}$ & $\mathbf{8}$ & The daily program at school is inopportune for me & .831 & 3.02 & middle \\
\hline $\mathbf{3}$ & $\mathbf{9}$ & $\begin{array}{l}\text { There are different books and resource in sign language for additionally } \\
\text { reading. }\end{array}$ & .815 & 2.99 & middle \\
\hline $\mathbf{1 6}$ & $\mathbf{1 0}$ & $\begin{array}{l}\text { in the school, there are programs of Integrating Hearing -impaired } \\
\text { students into the community }\end{array}$ & .941 & 2.97 & middle \\
\hline $\mathbf{7}$ & $\mathbf{1 1}$ & There are focusing on some activities more than others. & .929 & 2.94 & middle \\
\hline $\mathbf{2}$ & $\mathbf{1 2}$ & I'm involved in poetic, musical or dramatic activities at school events & .848 & 2.92 & middle \\
\hline $\mathbf{6}$ & $\mathbf{1 3}$ & I'm involved in the recreational activities at school celebrations & .792 & 2.90 & middle \\
\hline $\mathbf{1}$ & $\mathbf{1 4}$ & There are integrated recreational activities at school & .905 & 2.86 & middle \\
\hline $\mathbf{1 3}$ & $\mathbf{1 5}$ & The psychological counselor treatment was comfortable while asking him & .989 & 2.75 & middle \\
\hline $\mathbf{2 4}$ & $\mathbf{1 6}$ & I have a feeling of comfort while staying in school. & .942 & 2.65 & middle \\
\hline $\mathbf{2 0}$ & $\mathbf{1 7}$ & I am taking advantage of sports activities at school. & .919 & 2.61 & middle \\
\hline
\end{tabular}




\begin{tabular}{|c|c|c|c|c|c|}
\hline No & rank & Statements & $\begin{array}{c}\text { standard } \\
\text { deviations }\end{array}$ & Means & level \\
\hline 21 & 18 & I have feeling bored while playing sport daily & .914 & 2.59 & middle \\
\hline 22 & 19 & the time of sports activities suitable & .894 & 2.56 & middle \\
\hline 18 & 20 & The school cooperates with other institutions in multi activities & 1.022 & 2.51 & middle \\
\hline 25 & 21 & my will become stronger to change my life to better & .988 & 2.49 & middle \\
\hline 11 & 22 & $\begin{array}{l}\text { The school staff recognizes the importance of our social and psychological } \\
\text { care. }\end{array}$ & 1.024 & 2.41 & middle \\
\hline 10 & 23 & Developing the social ability of students to be effective members of society & .940 & 2.39 & middle \\
\hline 14 & 24 & $\begin{array}{l}\text { The school provides facilities, educational equipment ,and transportation } \\
\text { to meet the needs of their students }\end{array}$ & .768 & 2.35 & middle \\
\hline 15 & 25 & I need someone who I trust and tell him my problems in school & .833 & 2.13 & Low \\
\hline 12 & 26 & I talk to school staff with satisfaction & .874 & 2.03 & Low \\
\hline \multirow[t]{2}{*}{17} & 27 & I am taking advantage of recreational activities at school. & .945 & 2.01 & Low \\
\hline & & The means of coping strategies at school & .365 & 2.77 & middle \\
\hline
\end{tabular}

In this table, 2.77 was the overall mean of coping strategies of the Hearing -impaired students problems in deaf schools, while the means and standard deviations of the statements ranged between 2.01 and 2.42.

"The school protects students from violence and abuse." statement was the highest mean (3.42), while "I am taking advantage of recreational activities at school" was the lowest (2.77).

3.4 Are there any statistically significant differences among hearing-impaired based on (gender, place of residence, severity of disability, and school grade)?

To answer this question, descriptive statistics (means, standard deviations) and the T-test were calculated to investigate the Hearing -impaired students' responses to social and psychological problems and the coping strategies according to the gender, place of residence, the severity of the disability, and the grade.

The result showed that there are no statistically significant differences in Hearing -impaired students' responses due to gender, place of residence, stage of study and severity of the disability.

\section{Discussion}

\subsection{Discussion of the results of the first question:}

What are the social problems of hearing-impaired students?"

The results demonstrate that the social problems experienced by students with hearing disabilities show that there is difficulty in managing public places and fear of being subject to violence by others. This explains that social problems appear to them as a result of different ways of communicating with other people who listen to society, and their fear and lack of confidence in them as a result of different ways of communication between them due to the ways of communication between them in terms of sign language and mental abilities, and thus social problems are not apparent within their community unless they are communicated with the community of listeners. The results of this study are consistent with the results of the study by: (Watson, Neuman, Dickinson, 2001) Obstacle (2007) Karen, Kathryn, Blair, 2008) and Nta. The result of (Rimawi, 2008) study showed there are hearing and social difficulties facing the hearing impaired, and they appear at above average levels, especially anxiety levels.

\subsection{Discussion of the results of the Second question:}

What are the psychological problems of hearing-impaired students? 
The results showed that the psychological problems experienced by students with hearing disabilities were appearing in the form of psychological disorders, the most prominent of which was the resort to justification when mistakes were made, fear of making mistakes and feeling anxious when separated from close people, and was considered as a type of strategy used to prove one's self in a way that may appear wrong, such as anxiety and frustration, which turn into aggression, excessive activity and lack of school discipline as a result of repeated attempts of failure and repeated frustration. This explains that the sign language has a very important impact on the lives of students with hearing impairment. Their inability to communicate with their peers of hearers has negatively impacted their social adaptation to what is around them, which leads to the emergence of psychological problems; they are motivated to deal with situations in which they are exposed negatively. The results of this study are consistent with the study of the shoulders (2007) and the results of the study by (Shirin. Antia, Patricia, Luckner, 2011) that showed the presence of psychological, social and behavioral problems in the deaf and hearing - impaired to a moderate degree.

\subsection{Discussion of the results of the third question:}

"What is the adoption level of coping strategies for social and psychological problems in Deaf school?"

The results of this question showed that the level of strategies used to confront social and psychological problems in the deaf school was at an average level and it can be explained that the level of ownership of workers with deaf students from parents and teachers is not at a sufficient level to deal with the needs of deaf students with regard to their ability to meet their social and psychological needs, which Problems occur for students with hearing disabilities, which requires strategies that limit the problems of these students. This is consistent with the results of previous studies that examined special strategies which limit the problems of the hearing impaired, such as: Kare n, Kathryn, Blair, 2008) and (Abu Deraie, Al-Ramamneh, 2019).

\subsection{Discussion of the results of the Fourth question:}

Are there any statistically significant differences among hearing -impaired based on (gender, place of residence, severity of disability, and school grade)?

The results of this question showed that there are no statistically significant differences in social and psychological problems and strategies for facing social and psychological problems depending on the gender variable, place of residence, school stage, and severity of disability. This explains that all students with hearing disabilities with their different bicycles have the same behavioral and emotional characteristics that are reflected on aspects of their personality due to the absence of auditory communication and their feeling that they are different from their normal peers of the same age group, which makes them vulnerable to social and psychological disorders. This explains that deaf is a heterogeneous class, but they agree with each other with a set of characteristics, which are: sign language, total communication, the surrounding environment, activities and cultural clubs. Thus, this common diversity among them led to the acquisition of a culture of unified nature and thus psychological and social problems, if any. They are of the same nature and degree. This indicates that the study members with hearing disabilities have equal psychological problems according to their gender, age and degree of hearing impairment for them. The results of this question are consistent with the results of each of the studies by (Abu Drai, Al-Ramamneh, 2019).

\section{Recommendations}

Based on the findings, three recommendations are suggested. First, it is recommended to increase the awareness of the challenge that hearing-impaired students face. It is hoped that the findings will be 
shared with those who deal with hearing-impaired students. Second, it is necessary to increase inclusion conditions of hearing-impaired students with their hearing peers in schools to help reduce stereotypes and make them feel inclusive in the general student population. Finally, it is recommended to provide psychological clinical services to reduce the behavioral disorder associated with hearing loss.

\section{References}

Abu Dreea, S., \& Arramamneh, A. (2019). Performanca and Verbal intelligence and its relation to behavioral problems of hearing impaired students. The Jordan Journal of Applied science, (accepted for publishing),1-14.

AbuDreea, S. (2017). Standardization A Jordanian Version of The Wechsler Intelligence Scale for Children -Fourth Edition Adapted for Deaf Via Sign Language From 6-11,16 years. Phd dissertation.Amman, Jordan: The Word Islamic Science and Education University.

AbuSha'ban, A. (2016). Behavioral problems among hearing impaired and normal children in Gaza strip. Mater thesis, Gaza, Palestine: Islamic University.193.

AlA'iq, m., \& Yahya, K. (2007). Problems of Hearing Impaired students from thier teachers and famillies' perspectives. Master Thesis, Amman: Jordan University.126.

Ali, D. (2015). Social competence and its relation to social isolation in deaf. Scientific Research Journal in Education, 2(16), 113 - 136. doi:10.21608/JSRE.2015.14362

Alrousan, F. (2016). Diagnosis and measurement methods in special education (7th ed.). Amman, Jordan: Daralfiker Publishers and Distrbuitors.

ALzahrani, A. (2007). Modern trends in verbal learning for deaf and hearing- impaired concepts, principles, and practice based on. Special Education Conference (pp. 1120-1085). Egypt: Benha University.

Hay, I. ( 2005 ). Facilitating children's self-concept: A rationale and evaluative study .Australian Journal of Guidance \& Counselling,15,60 - 67. Doi:10.1375 / ajgc . 15 .1.6o.

Karen, L., Kathryn, F \& B Bair ,D (2008). Communication disorders in the school: Perspectives on academic and social success an introduction. Psychology in the Schools. Hyperlink "https://onlinelibrary.wiley.com/toc/15206807/2008/45/7" 45( 7 ). 579-581.

Naji, S., \& Qurashi, A. (2018, March). The behvioural diorder of deaf children. El-Bahith Journal in Social Science and Humanities 10(33), 741-749.

Rimawi, A. M. (2008). The Ssocial/emotional difficulties facing the disabled women and their relation with disability category. DIRASAT-Educational Science, 35 (appendix), 558 - 578.

Shirin, D. A., Patricia J., John, L. (2011). Social Outcomes of Students Who are Deaf and Hard of Hearing in General Education Classrooms. Exceptional Children . 77(4),1-15.

Vanaldik, T., Treffers, Veerman,J.,\& Yerhulst,F(2004) Mental health problems Of deaf Dutch children as indicated by parents ,responses to the children. Behavior Checklist, American Annals of the Deaf.148:390-395.

Watson, R., Neuman, S., \& Dickinson, D.(2001) Literacy and oral language: Implications for early literacy acquisition, Handbook of early literacy research : Volume 1, New York The Guilford Press (pg. 43-53). 